\title{
Combination of bevacizumab and photodynamic therapy vs. bevacizumab monotherapy for the treatment of wet age-related macular degeneration: A meta-analysis of randomized controlled trials
}

\author{
QINGQUAN WEI $^{1}$, JUNLING LIU $^{1}$, QINGYU LIU $^{1}$, CHENGDA REN $^{1}$, \\ WENTING CAI ${ }^{1}$, XIUWEI LIANG ${ }^{1}$, JING WEN $^{2}$ and JING YU ${ }^{1,3}$

\begin{abstract}
Departments of ${ }^{1}$ Ophthalmology and ${ }^{2}$ Education, Shanghai Tenth People's Hospital Affiliated with Tongji University, Shanghai 200072; ${ }^{3}$ Department of Ophthalmology, Ninghai First Hospital, Ninghai, Zhejiang 315600, P.R. China
\end{abstract}

Received December 23, 2017; Accepted June 1, 2018

DOI: $10.3892 /$ etm.2018.6305

\begin{abstract}
The purpose of this meta-analysis was to compare the efficacy and safety of the combination of bevacizumab and photodynamic therapy (PDT) with bevacizumab monotherapy for the treatment of age-related macular degeneration (AMD). Patients with active choroidal neovascularization (CNV) secondary to AMD were included in the present study. The treatment group included patients treated with a combination of bevacizumab and PDT and patients treated with bevacizumab monotherapy. Only randomized controlled trials (RCTs) were included in the analysis. The PubMed, EMBASE and Cochrane Central Register of Controlled Trials databases were searched. Meta-analysis was performed using RevMan v.5.3 software, and best-corrected visual acuity (BCVA), central retinal thickness (CRT) and the average number of bevacizumab retreatments were assessed. A total of 5 RCTs were included in the analysis. There were no significant differences observed in the mean BCVA change between the combination treatment group and the bevacizumab monotherapy group [standard mean difference $0.20 ; 95 \%$ confidence interval (CI) $-0.53,0.93, \mathrm{P}=0.59]$. There were also no significant differences in the CRT increases between the two groups [weighted mean difference (WMD) $-22.16,95 \% \mathrm{CI}-52.01$ to $7.69, \mathrm{P}=0.15]$. No significant differences were observed in the proportions of
\end{abstract}

Correspondence to: Dr Jing Wen, Department of Education, Shanghai Tenth People's Hospital Affiliated with Tongji University, 301 Middle Yanchang Road, Shanghai 200072, P.R. China E-mail: 13764341057@163.com

Dr Jing Yu, Department of Ophthalmology, Shanghai Tenth People's Hospital Affiliated with Tongji University, 301 Middle Yanchang Road, Shanghai 200072, P.R. China

E-mail: dryujing@aliyun.com

Key words: meta-analysis, bevacizumab, photodynamic therapy, age-related macular degeneration, treatment patients gaining $>15$ letters between the two groups [risk ratio (RR) 0.86 , 95\% CI $0.64,1.15, \mathrm{P}=0.30]$. However, the average number of the ranibizumab retreatments was significantly lower in the combination treatment group compared with the bevacizumab monotherapy group (WMD, -2.70, 95\% CI -3.93 to $-1.46 ; \mathrm{P}<0.0001)$. Additionally, there were no significant differences in the rate of ocular adverse events (RR, 0.57; $95 \% \mathrm{CI}, 0.27$ to $1.22 ; \mathrm{P}=0.15$ ) and systemic adverse events ( $R R$, 5.42; $95 \% \mathrm{CI}, 0.29$ to $101.77 ; \mathrm{P}=0.26$ ) between the two groups. In conclusion there were no significant differences in mean BCVA change, CRT increases, the proportions of patients gaining $>15$ letters, or the incidences of ocular adverse events and systemic adverse events. However, combination treatment may significantly reduce the average number of bevacizumab retreatments compared with monotherapy.

\section{Introduction}

Age-related macular degeneration (AMD) is the leading cause of irreversible vision loss in people older than $50 \mathrm{y}$ of age in the developed world (1). Although the neovascular form accounts for only $20 \%$ of AMD cases, it is the main cause of severe vision loss in almost $90 \%$ of patients with $\operatorname{AMD}(2,3)$. Choroidal neovascularization (CNV) is the primary pathology underlying wet AMD and is a pathological form of neovascularization that can cause bleeding, hemorrhage, fibrosis, and retinal pigment epithelium and neurosensory functional damage, eventually resulting in vision loss (4).

At present, there are many treatments available for AMD. The most common treatments are verteporfin photodynamic therapy (PDT) and anti-vascular endothelial growth factor (VEGF) drugs administered via intraocular injection (5). Bevacizumab is a humanized anti-VEGF monoclonal IgG1 antibody that binds to all isoforms of VEGF and can therefore prevent angiogenesis (6). Off-label use of bevacizumab has been shown to be reasonably safe and very effective in the treatment of AMD (7-9). Several studies have shown that bevacizumab and ranibizumab have equivalent efficacy and comparable safety (10-15). Bevacizumab is still widely used 
for this off-label indication not only because it has a mode of action similar to that of ranibizumab but also because its cost is considerably lower than that of ranibizumab $(16,17)$. However, bevacizumab monotherapy requires multiple reinjections (18) and is associated with the risk of endophthalmitis, cataract formation and uveitis (19-23), as well as an increased risk of thromboembolic events (24).

PDT with verteporfin has historically been the standard treatment for AMD and has been shown to benefit patients affected by classic CNV in the setting of AMD (25-27). Combining PDT with bevacizumab may reduce the number of required treatments and preserve or even enhance visual acuity. Increasing numbers of studies have reported that PDT combined with intravitreal bevacizumab is an effective option for patients with AMD, as this combination facilitates improvements in visual acuity, decreases in central retinal thickness (CRT) and reductions in the number of retreatments (28-31). Studies comparing combination therapy with monotherapy have reported that combination therapy significantly improves visual acuity compared to monotherapy (32). However, the ideal maintenance regimen for this combination remains an area of scientific debate. Four clinical randomized controlled trials (RCTs) showed that there were no significant differences in visual gain between patients receiving the combination of PDT and bevacizumab and patients receiving monotherapy, although these studies found that the combination of the two agents reduced reinjection rates (30,33-35).

Therefore, we performed a meta-analysis of RCTs to compare the efficacy and safety of the combination of verteporfin PDT and intravitreal bevacizumab therapy with those of bevacizumab monotherapy in patients with AMD.

\section{Materials and methods}

Search strategy. A systematic English language search of PubMed, EMBASE and the Cochrane Central Register of Controlled Trials for human studies published up to October 2017 was conducted, with language restrictions. Key terms included AMD, bevacizumab, avastin and PDT. The search was restricted to RCTs. We manually searched the reference lists of all original studies and review articles identified by the electronic search to identify other potentially eligible articles.

Inclusion criteria. We selected the following studies: i) studies including patients with active CNV secondary to AMD; ii) studies featuring a randomized controlled trial (RCT) design comparing the combination of bevacizumab and PDT with bevacizumab monotherapy and iii) studies measuring at least one outcome of interest.

Exclusion criteria. The following studies were excluded: i) studies that were not RCTs; ii) studies of CNV not caused by AMD and iii) unpublished conference abstracts.

Data extraction and quality assessment. Titles and abstracts were reviewed by two reviewers using the above selection criteria. Full-text versions of all relevant studies were obtained for detailed evaluations. Data extraction and quality assessments were conducted using the modified Jadad assessment tool (36).
Disagreements were resolved via consensus after discussion. The following data were extracted from each study: the name of the first author, the study design, and the major inclusion and exclusion criteria, as well as information regarding study population characteristics (age, sex, no. of eyes in the study), intervention groups, follow-up durations and outcomes (ocular and systemic adverse effects). Data regarding changes in best-corrected visual acuity (BCVA), the numbers of patients with gains of more than 15 letters, the average numbers of bevacizumab retreatments, and changes in CRT were also extracted.

Statistical analysis. The meta-analysis was conducted using RevMan v.5.3 software. Risk ratios (RRs) were measured using 95\% confidence intervals (CIs) for dichotomous data, while weighted mean differences (WMDs) were measured using 95\% CIs for continuous data. Standard mean differences (SMDs) were used when all the trials assessed the same outcomes in a variety of ways. The Q test or $\mathrm{I}^{2}$ test was used to evaluate heterogeneity. An $\mathrm{I}^{2}$ value of $>50 \%$ accompanied with a P-value $<0.05$ for the $\mathrm{Q}$ test was determined to indicate the presence of significant heterogeneity. Both fixed-effects and random-effects models were used to obtain summary RRs, WMDs or SMDs. In the absence of heterogeneity between groups, the fixed-effects model and random-effects model yielded concordant results. When heterogeneity was significant, the random effects model was employed. Potential publication bias was estimated using the Egger test and by visually evaluating a funnel plot.

\section{Results}

Literature search. A flow chart of the selection process used to identify eligible studies is shown in Fig. 1. A total of 205 articles were initially identified. After duplicates were screened for potentially relevant articles, 194 articles were deemed eligible for further evaluation. We screened the titles and abstracts of these articles and identified 6 eligible studies. We subsequently read the text of each article and found 1 from the same study group, which was excluded. Ultimately, five published (30,32-35) articles were eligible for analysis.

Included studies. The basic characteristics of the five included studies are shown in Table I. Sample sizes ranged from 23 to 106 eyes. Mean patient ages ranged from 63.2 to 83.4 years. The dose of bevacizumab was $1.25 \mathrm{mg}$ in the bevacizumab monotherapy groups of the included studies. The doses of verteporfin PDT and bevacizumab were $25 \mathrm{~J} / \mathrm{cm}^{2}$ standard fluence (SF) and $1.25 \mathrm{mg}$, respectively, in the combination therapy groups of the included studies, with the exception of the Lazic and Gabric (32) study, in which the dose of PDT was not mentioned. Moreover, the duration of follow-up varied from 3 to 12 months among the studies. The five studies were assessed regarding methodological quality, according to the Jadad score and were determined to be of high quality.

\section{Estimation of outcomes}

Changes in mean BCVA compared with baseline. Visual acuity was the most important outcome measure with respect to treatment efficacy. The results regarding changes in mean BCVA are shown in Fig. 2A. There were no significant differences in changes in BCVA between the combination group and 


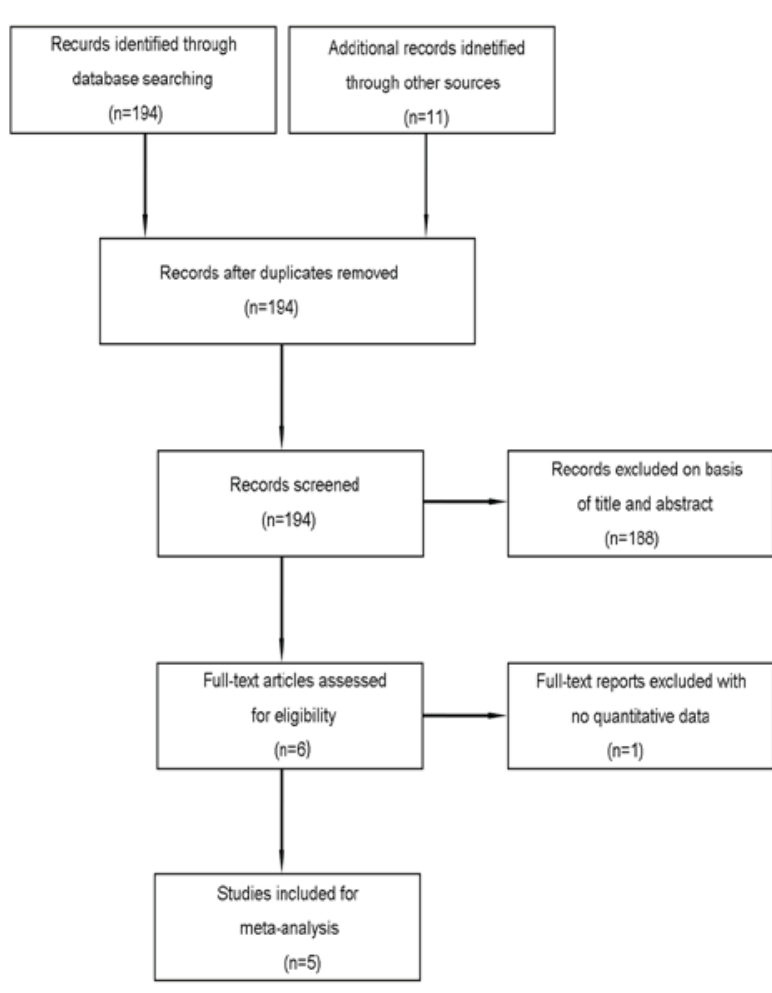

Figure 1. Study selection.

bevacizumab group (SMD 0.20; 95\% CI $-0.53,0.93, \mathrm{P}=0.59$ ). The random-effects model was used due to the high heterogeneity of the effect size $\left(I^{2}=91 \%, P<0.00001\right)$. The dose of PDT is not mentioned in Lazic and Gabric (32) study and this may have heterogeneous. So we removed the Lazic and Gabric (32) study to apply the sensitivity analysis and found that the result of statistical analysis was still insignificant (SMD, -0.11; 95\% CI -0.35, 0.13, P=0.39; Fig. 2B).

Number of patients who gained more than 15 letters. We extracted the number of patients who gained more than 15 letters. Because the data were not heterogeneous $\left(\mathrm{I}^{2}=0 \%, \mathrm{P}=0.78\right)$, the fixed-effects model was used. The pooled RR showed that there was no significant difference between the two intervention groups regarding the number of patients who gained more than 15 letters (RR 0.86, 95\% CI 0.64, 1.15, P=0.30; Fig. 3).

CRT. CRT is the most important anatomical change associated with AMD treatment. The effects of the combination of bevacizumab and PDT on CRT compared with those of bevacizumab monotherapy are shown in Fig. 4. The pooled results indicate that there was no significant difference between the two groups regarding changes in CRT (WMD $-22.16,95 \% \mathrm{CI}$ -52.01 to $7.69, \mathrm{P}=0.15$ ).

Average number of bevacizumab retreatments. Three studies measured the average number of bevacizumab retreatments. The pooled results indicate that the average number of bevacizumab retreatments was significantly lower in the combination therapy group than in the bevacizumab monotherapy group (WMD $-2.70,95 \%$ CI -3.93 to $-1.46, \mathrm{P}<0.0001$; Fig. 5). The random-effects model was used due to the high heterogeneity of the effect size $\left(\mathrm{I}^{2}=95 \%, \mathrm{P}<0.00001\right)$.
Adverse events. Four $(30,32,34,35)$ of five studies [excluding Datseris et al (33)] reported data regarding ocular adverse events and systematic adverse events. We compared the numbers of ocular adverse events and non-ocular adverse events in the combination group with those in the bevacizumab monotherapy group and noted that four were no significant differences between the two intervention groups with respect to those parameters (Fig. 6). All adverse events reported in the abovementioned three studies are shown in Table II.

Heterogeneity, sensitivity analysis, and publication bias. The dose of PDT is not mentioned in one study and this may have heterogeneous. After excluding the study, the analysis results not changed. A sensitivity analysis was conducted to assess the stability of the results by sequential removal of individual studies. When the analysis result is high heterogeneity, we use random effects model. These sensitivity analyses indicated that our conclusions were generally robust. Funnel plots and Egger test were not used because there were less than ten studies for each comparison.

\section{Discussion}

In this meta-analysis, we assessed four RCTs including 371 patients (183 patients in the combination group and 188 patients in the bevacizumab group). Lazic and Gabric (32), noted significant improvements in BCVA at 3 months after combination therapy with verteporfin PDT and intravitreal bevacizumab. Some studies observed that there was no significant differences in visual acuity improvement between the bevacizumab monotherapy group and combination therapy group (30,33-35). The results of our meta-analysis indicated that the bevacizumab monotherapy group experienced improvements in BCVA similar to those of the combination therapy group, indicating that the efficacies of the two therapy regimens were similar with respect to this parameter. We assessed the numbers of patients who gained more than 15 letters and determined that there was no significant difference between two groups with respect to this parameter. Regarding mean changes in CRT, bevacizumab monotherapy demonstrated efficacy equivalent to that of combination therapy. PDT does not have a destructive impact on patient vision, as is the case with older treatments, and stabilizes wet AMD progression (37). However, the combination of PDT and bevacizumab can result in more rapid and permanent $\mathrm{CNV}$ occlusion (8), resulting in increased ocular VEGF levels (38). These findings may explain the similar efficacies exhibited by the two treatment regimens.

Reinjection rates were significantly lower in patients treated with combination therapy than in patients treated with bevacizumab monotherapy. In this meta-analysis, we noted that the average number of bevacizumab reinjections in the combination group was lower than that in the bevacizumab monotherapy group. These findings support the hypothesis that combination treatment exerts synergistic effects, resulting in a reduced need for subsequent injections compared with monotherapy. Thus, combination therapy may be a more cost-effective option than monotherapy for the treatment of neovascular AMD.

The included RCTs indicated that both treatments were safe. The majority of adverse events associated with 


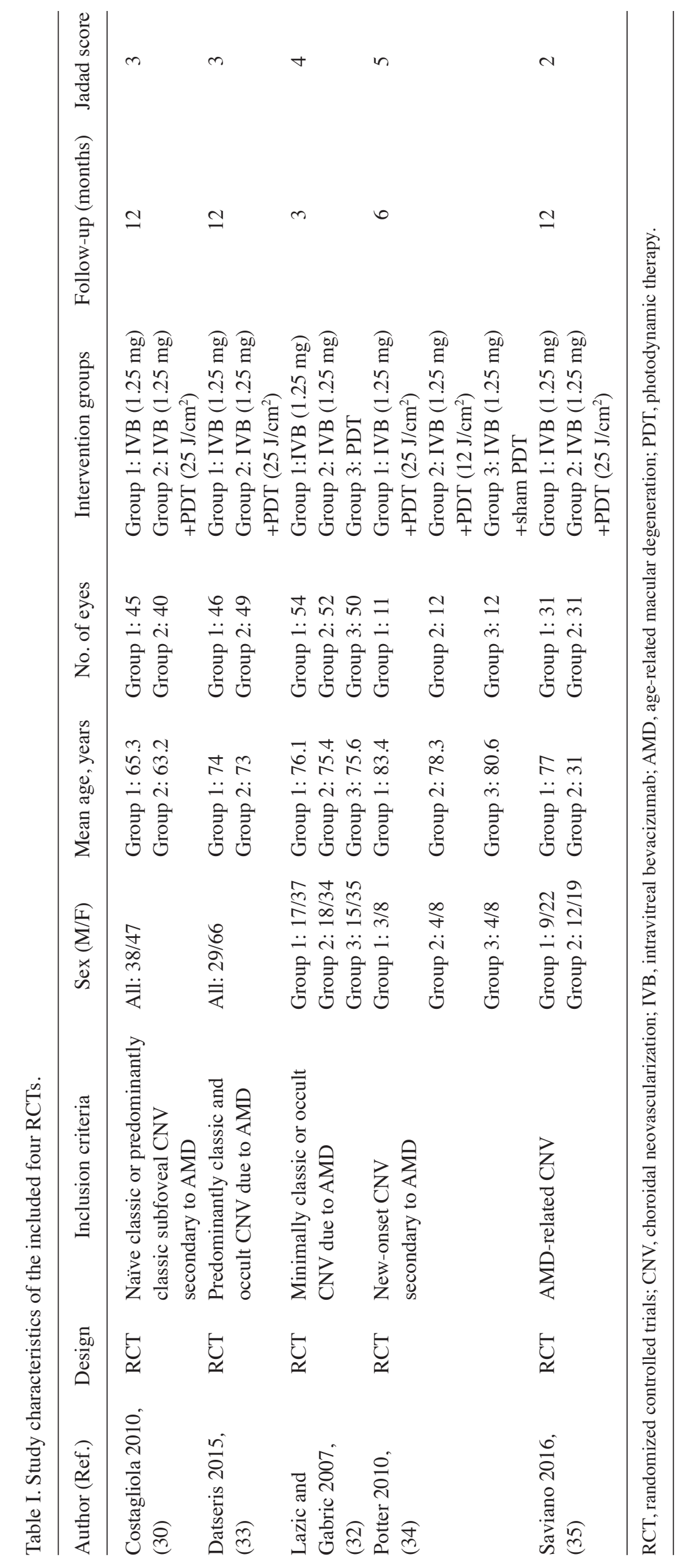


Combination Bevacizumab

Std. mean difference

Std. mean difference

Study or Subgroup Mean SD Total Mean SD Total Weight IV, Random, 95\% C

IV, Random, $95 \% \mathrm{Cl}$

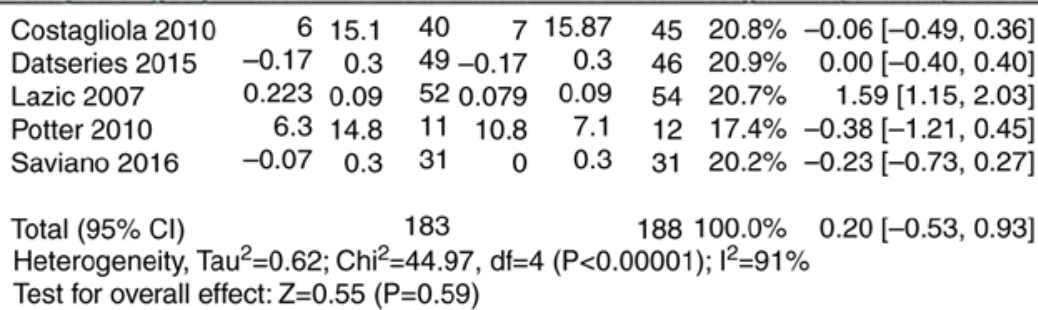

B

Combination Bevacizumab

Std. mean difference

Study or Subgroup Mean SD Total Mean SD Total Weight IV, Fixed, $95 \% \mathrm{Cl}$

\begin{tabular}{|c|c|c|c|c|c|c|}
\hline Costagliola 2010 & 6 & 15.1 & 40 & 15.87 & 45 & $32.1 \%-0.06[-0.49,0.36]$ \\
\hline Datseries 2015 & -0.17 & 0.3 & $49-0.17$ & 0.3 & 46 & $36.0 \% \quad 0.00[-0.40,0.40]$ \\
\hline Potte & 6.3 & 14.8 & 1110.8 & 7.1 & 12 & $8.5 \%-0.38[-1.21,0.45]$ \\
\hline Savi & -0.07 & 0.3 & 31 & 0.3 & 31 & $23.4 \%-0.23[-0.73,0.27]$ \\
\hline Total ( & & & 131 & & 134 & $00.0 \% \quad 0.11[-0.35,0.27]$ \\
\hline
\end{tabular}

Heterogeneity, $\mathrm{Chi}^{2}=0.96 ; \mathrm{df}=3(\mathrm{P}=0.81) ; \mathrm{I}^{2}=0 \%$

Test for overall effect: $Z=0.87(P=0.39)$

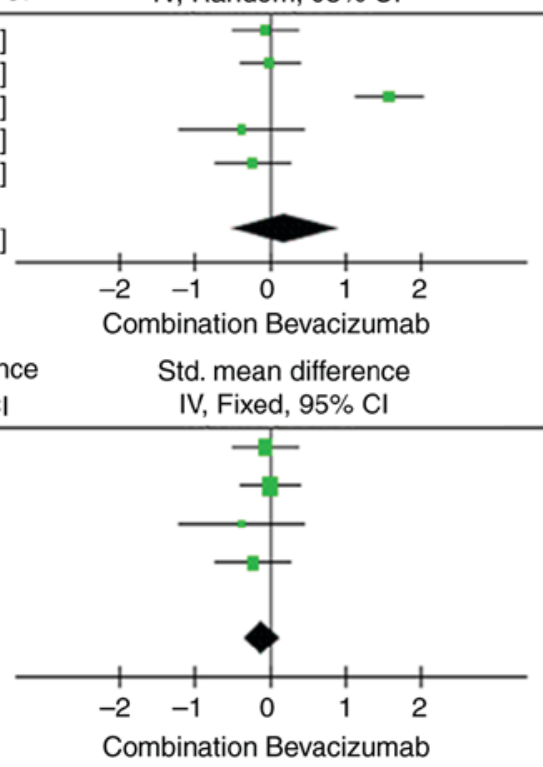

Figure 2. Forest plot showing the changes in mean BCVA compared with the baseline. (A) The Lazic and Gabric (32) study included. (B) The Lazic and Gabric (32) study excluded. BCVA, best-corrected visual acuity; SD, standard deviation; CI, confidence interval.

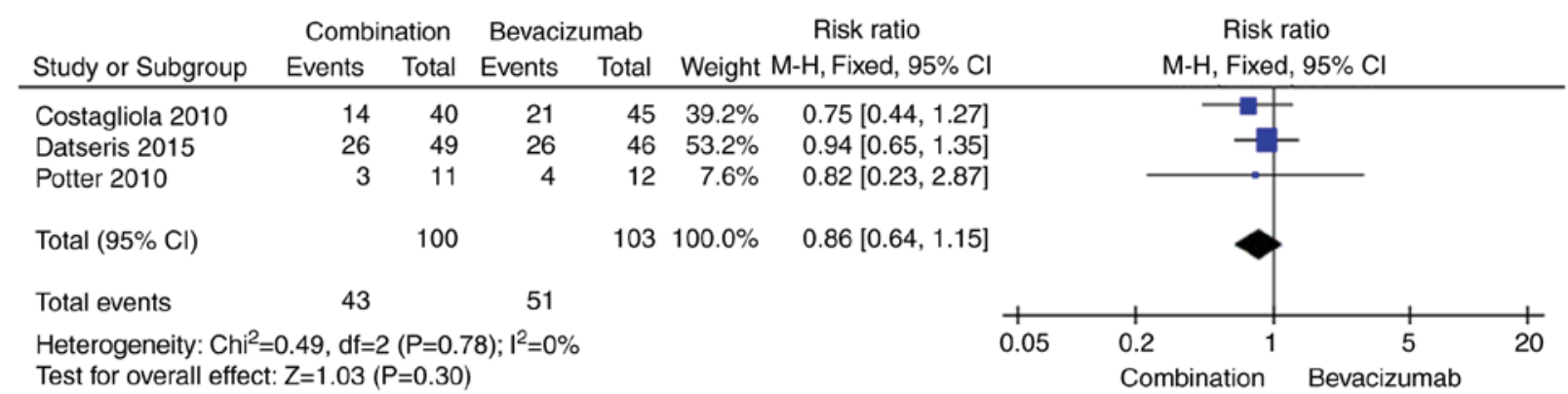

Figure 3. Forest plot showing the number of patients who gained $>15$ letters compared with the baseline. SD, standard deviation; CI, confidence interval.

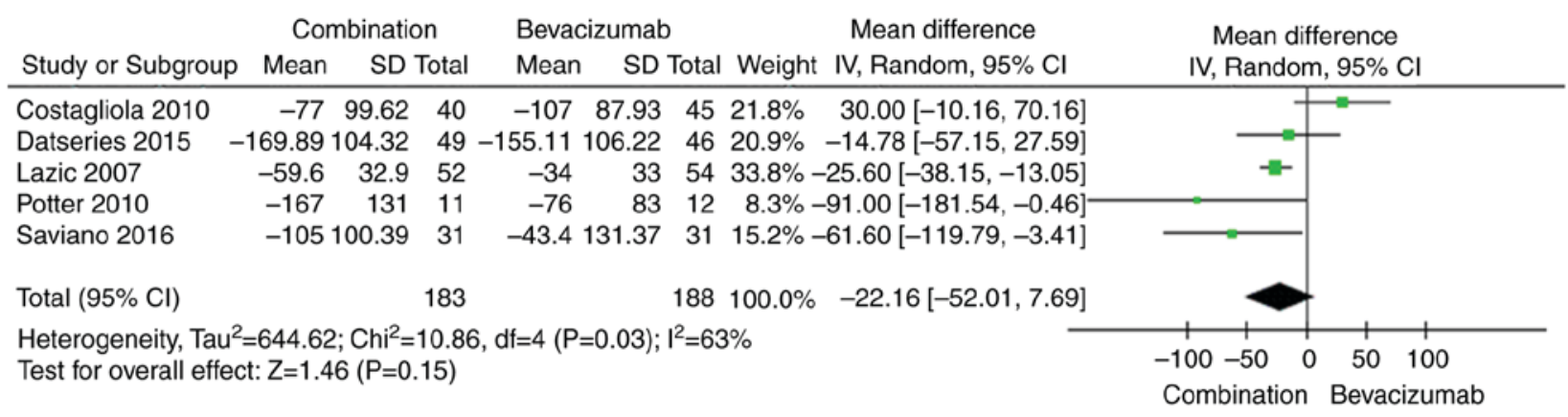

Figure 4. Forest plot showing the changes in mean CRT compared with the baseline. CRT, central retinal thickness; SD, standard deviation; CI, confidence interval.

bevacizumab monotherapy and combination treatment were of moderate severity. No serious adverse events, such as death or endophthalmitis, were noted in any of the included RCTs. One patient died of a stroke (34). Ocular adverse events occurred more frequently in the bevacizumab monotherapy group than in the combination therapy group, most likely due to the use of intravitreal injections in the former group. The most significant side effects associated with the two treatments were posterior vitreous detachments and cataracts. Other side effects, such as increased anterior chamber cell pigment epithelium tears and vision loss of more than 20 letters, were reported in three studies. However, there was no difference in the incidence of ocular adverse events between the two groups. Systemic adverse events, such as hypertension and myocardial infraction, were reported only in the combination therapy group of one study (34). We noted no significant difference in 
Table II. Main ocular adverse events and systemic adverse events reported in the three studies.

\begin{tabular}{|c|c|c|c|c|c|c|}
\hline \multirow[b]{2}{*}{ Side effects } & \multicolumn{3}{|c|}{ Combination } & \multicolumn{3}{|c|}{ Bevacizumab } \\
\hline & Events & Total & Incidence (\%) & Events & Total & Incidence $(\%)$ \\
\hline \multicolumn{7}{|l|}{ Ocular adverse events } \\
\hline Pigment epithelium tears & 0 & 103 & 0 & 3 & 111 & 2.7 \\
\hline Posterior vitreous detachments & 4 & 103 & 3.9 & 8 & 111 & 7.2 \\
\hline Cataract progressions & 3 & 103 & 2.9 & 4 & 111 & 3.6 \\
\hline Vision loss $\geq 20$ letters & 1 & 103 & 1 & 0 & 111 & 0 \\
\hline Non-ocular adverse events & 1 & 103 & 1 & 0 & 111 & 0 \\
\hline Hypertension & 1 & 103 & 1 & 0 & 111 & 0 \\
\hline Myocardial infraction & 1 & 103 & 1 & 0 & 111 & 0 \\
\hline Mortality $^{\mathrm{a}}$ & & & & & & \\
\hline
\end{tabular}

${ }^{\mathrm{a}}$ One subject had a stroke and succumbed to mortality.

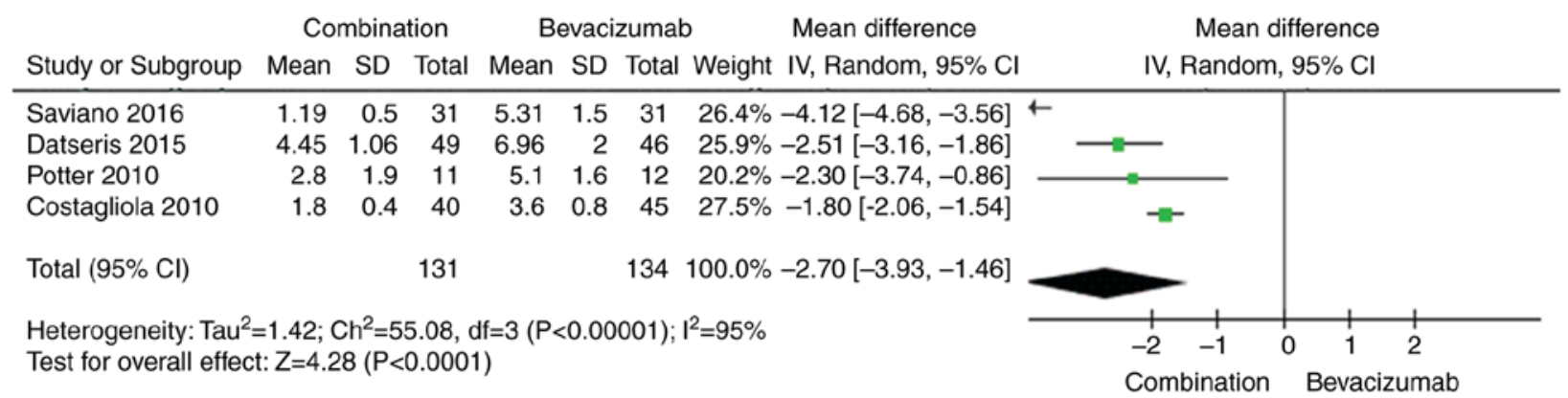

Figure 5. Forest plot showing the number of bevacizumab retreatments. SD, standard deviation; CI, confidence interval.

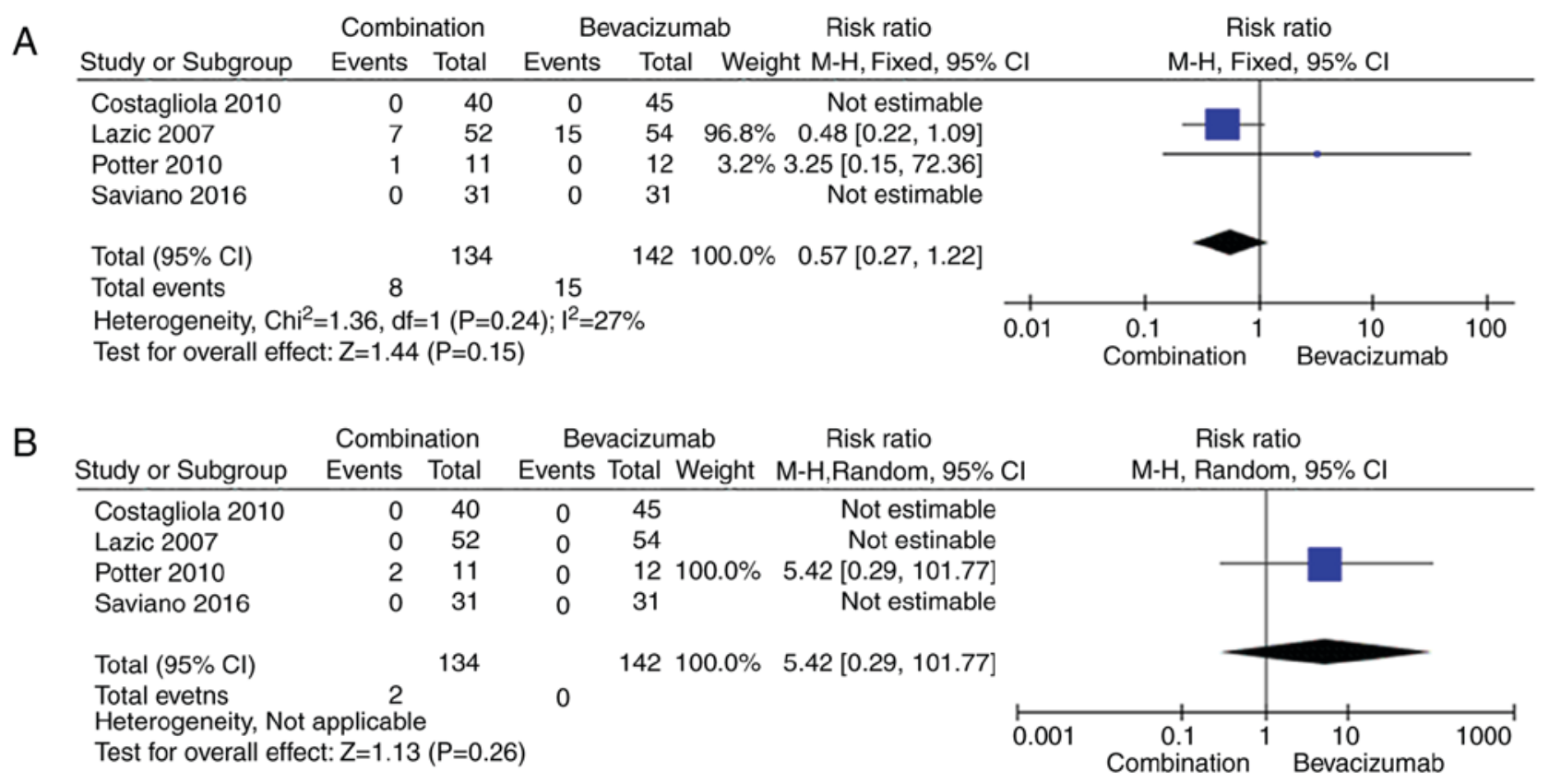

Figure 6. Forest plot showing the number of ocular adverse events and systemic adverse events. (A) Ocular adverse events. (B) Systemic adverse events.

the incidence of adverse events between the two groups, findings consistent with those of related clinical trials. However, as the number of studies included in our analysis was small, additional RCTs comparing the efficacy and safety of bevacizumab monotherapy and combination therapy among larger groups of patients are necessary to confirm our findings. 
This meta-analysis had some limitations. Verteporfin PDT has been shown to be more effective at treating classic choroidal neovascularization than mild or classic occult $\mathrm{CNV}$, and this analysis did not examine its efficacy with respect to the different types of CNV. Thus, additional studies are required. Furthermore, the differences in the durations of the included trials ( 3 to 12 months) were a potential source of heterogeneity. Some studies did not provide means and standard deviations, electing to report only before- and after-treatment values or medians and ranges, which may have resulted in data conversion-related errors. Finally, the numbers of bevacizumab treatments administered in the included trials were not uniform, as the average numbers of bevacizumab injections differed among the studies. Additionally, the dose of PDT was not mentioned in one of the included studies, which have may have biased our results. And the small number of studies included in this meta-analysis was a limitation of the study, and that publication bias could therefore not be assessed.

In conclusion, there were no significant differences in mean BCVA changes, CRT increases, the proportions of patients gaining more than 15 letters, or the incidences of ocular adverse events and systemic adverse events between the two groups. However, combination therapy may significantly reduce the average number of bevacizumab retreatments compared with monotherapy. And this systematic review and meta-analysis may provide a basis for clinical treatment of wet AMD.

\section{Acknowledgements}

Not applicable.

\section{Funding}

The present study was financially supported by the National Natural Science Foundation of China (grant no. 81470648).

\section{Availability of data and materials}

All data generated or analyzed during this study are included in this published article.

\section{Authors' contributions}

JY and JW designed the study. QW, JL and QL screened the literature. $\mathrm{CR}, \mathrm{WC}$ and $\mathrm{XL}$ extracted the data from the literature. QW, JL, JY and JW conducted the meta-analysis and wrote the manuscript. JY and JW submitted the study.

\section{Ethics approval and consent to participate}

Not applicable.

\section{Consent for publication}

Not applicable.

\section{Competing interests}

The authors declare that they have no competing interests.

\section{References}

1. Bressler NM: Age-related macular degeneration is the leading cause of blindness. JAMA 291: 1900-1901, 2004.

2. Position of the Retinological Society, the German Ophthalmological Society and the Professional Association of Ophthalmologists in Germany on the current therapeutic possibilities for neovascular age-related macular degeneration. Klin Monbl Augenheilkd 224: 559-566, 2007.

3. Aiello LP, Northrup JM, Keyt BA, Takagi H and Iwamoto MA: Hypoxic regulation of vascular endothelial growth factor in retinal cells. Arch Ophthalmol 113: 1538-1544, 1995.

4. Buch H, Vinding T and Nielsen NV: Prevalence and causes of visual impairment according to World Health Organization and United States criteria in an aged, urban Scandinavian population: The Copenhagen City Eye Study. Ophthalmology 108: 2347-2357, 2001.

5. Mitchell P, Annemans L, White R, Gallagher M and Thomas S: Cost effectiveness of treatments for wet age-related macular degeneration. Pharmacocconomics 29: 107-131, 2011.

6. Shao J, Choudhary MM and Schachat AP: Neovascular age-related macular degeneration. Dev Ophthalmol 55: 125-136, 2016.

7. EI-Mollayess GM, Noureddine BN and Bashshur ZF: Bevacizumab and neovascular age related macular degeneration: Pathogenesis and treatment. Semin Ophthalmol 26: 69-76, 2011.

8. Leydolt C, Michels S, Prager F, Garhoefer G, Georgopoulos M, Polak K and Schmidt-Erfurth U: Effect of intravitreal bevacizumab (Avastin) in neovascular age-related macular degeneration using a treatment regimen based on optical coherence tomography: 6- and 12-month results. Acta Ophthalmol 88: 594-600, 2010.

9. Takahashi M, Sato T and Kishi S: Intravitreal bevacizumab for age-related macular degeneration with good visual acuity. Jpn J Ophthalmol 54: 565-570, 2010.

10. Berg K, Hadzalic E, Gjertsen I, Forsaa V, Berger LH, Kinge B, Henschien H, Fossen K, Markovic S, Pedersen TR, et al: Ranibizumab or bevacizumab for neovascular age-related macular degeneration according to the lucentis compared to avastin study treat-and-extend protocol: Two-year results. Ophthalmology 123: 51-59, 2016.

11. Berg K, Pedersen TR, Sandvik L and Bragadóttir R: Comparison of ranibizumab and bevacizumab for neovascular age-related macular degeneration according to LUCAS treat-and-extend protocol. Ophthalmology 122: 146-152, 2015.

12. Moja L, Lucenteforte E, Kwag KH, Bertele V, Campomori A, Chakravarthy U, D'Amico R, Dickersin K, Kodjikian L, Lindsley K, et al: Systemic safety of bevacizumab versus ranibizumab for neovascular age-related macular degeneration. Cochrane Database Syst Rev 15: CD011230, 2014.

13. Chen G, Li W, Tzekov R, Jiang F, Mao S and Tong Y: Bevacizumab versus ranibizumab for neovascular age-related macular degeneration: A meta-analysis of randomized controlled trials. Retina 35: 187-193, 2015.

14. Kodjikian L, Decullier E, Souied EH, Girmens JF, Durand EE, Chapuis FR and Huot L: Bevacizumab and ranibizumab for neovascular age-related macular degeneration: An updated meta-analysis of randomised clinical trials. Graefes Arch Clin Exp Ophthalmol 252: 1529-1537, 2014.

15. Wang W and Zhang X: Systemic adverse events after intravitreal bevacizumab versus ranibizumab for age-related macular degeneration: A meta-analysis. PLoS One 9: e109744, 2014.

16. Solomon SD, Lindsley KB, Krzystolik MG, Vedula SS and Hawkins BS: Intravitreal bevacizumab versus ranibizumab for treatment of neovascular age-related macular degeneration: Findings from a cochrane systematic review. Ophthalmology 123: 70-77.e1, 2016.

17. Stein JD, Newman-Casey PA, Mrinalini T, Lee PP and Hutton DW: Cost-effectiveness of bevacizumab and ranibizumab for newly diagnosed neovascular macular degeneration. Ophthalmology 121: 936-945, 2014.

18. Fong KC, Kirkpatrick N, Mohamed Q and Johnston RL: Intravitreal bevacizumab (Avastin) for neovascular age-related macular degeneration using a variable frequency regimen in eyes with no previous treatment. Clin Experiment Ophthalmol 36: 748-755, 2008.

19. Kanchanaranya N, Rojdamrongratana D and Piyasoonthorn P: Incidence of post-intravitreal anti-VEGF endophthalmitis at Thammasat University Hospital. J Med Assoc Thai 98: 489-494, 2015.

20. Haddock LJ, Ramsey DJ and Young LH: Complications of subspecialty ophthalmic care: Endophthalmitis after intravitreal injections of anti-vascular endothelial growth factor medications. Semin Ophthalmol 29: 257-262, 2014. 
21. Lyall DA, Tey A, Foot B, Roxburgh ST, Virdi M, Robertson C and MacEwen CJ: Post-intravitreal anti-VEGF endophthalmitis in the United Kingdom: Incidence, features, risk factors, and outcomes. Eye (Lond) 26: 1517-1526, 2012.

22. Stewart MW: Endophthalmitis after injections of anti-vascular endothelial growth factor drugs. Retina 31: 1981-1982, 2011.

23. Inoue M, Kobayakawa S, Sotozono C, Komori H, Tanaka K, Suda Y, Matsushima H, Kinoshita S, Senoo T, Tochikubo T and Kadonosono K: Evaluation of the incidence of endophthalmitis after intravitreal injection of anti-vascular endothelial growth factor. Ophthalmologica 226: 145-150, 2011.

24. Carneiro AM, Barthelmes D, Falcão MS, Mendonça LS, Fonseca SL, Gonçalves RM,Faria-Correia F and Falcão-Reis FM: Arterial thromboembolic events in patients with exudative age-related macular degeneration treated with intravitreal bevacizumab or ranibizumab. Ophthalmologica 225: 211-221, 2011.

25. Schmidt-Erfurth UM and Pruente C: Management of neovascular age-related macular degeneration. Prog Retin Eye Res 26: 437-451, 2007

26. Incorvaia C, Campa C, Parmeggiani F, Menzione M, D'Angelo S, Della Corte M, Rinaldi M, Romano M, Dell'omo R and Costagliola C: 12-month retrospective study and review of photodynamic therapy with verteporfin for subfoveal choroidal neovascularization in age-related macular degeneration. Retina 28: 289-297, 2008.

27. Yonekawa Y and Kim IK: Clinical characteristics and current treatment of age-related macular degeneration. Cold Spring Harb Perspect Med 5: a017178, 2014.

28. Ladewig MS, Karl SE, Hamelmann V, Helb HM, Scholl HP Holz FG and Eter N: Combined intravitreal bevacizumab and photodynamic therapy for neovascular age-related macular degeneration. Graefes Arch Clin Exp Ophthalmol 246: 17-25, 2008.

29. Kim HW, Kim JL, Lee MH, Yoo HG, Chung IY and Lee JE: Combined treatment of photodynamic therapy and bevacizumab for choroidal neovascularization secondary to age-related macular degeneration. Korean J Ophthalmol 25: 231-237, 2011

30. Costagliola C, Romano MR, Rinaldi M, dell'Omo R, Chiosi F, Menzione $\mathrm{M}$ and Semeraro F: Low fluence rate photodynamic therapy combined with intravitreal bevacizumab for neovascular age-related macular degeneration. Br J Ophthalmol 94: 180-184, 2010.

31. Kaiser PK; Registry of Visudyne AMD Therapy Writing Committee, Boyer DS, Garcia R, Hao Y, Hughes MS, Jabbour NM, Kaiser PK, Mieler W, Slakter JS, et al: Verteporfin photodynamic therapy combined with intravitreal bevacizumab for neovascular age-related macular degeneration. Ophthalmology 116: 747-755, 755.e1, 2009
32. Lazic R and Gabric N: Verteporfin therapy and intravitreal bevacizumab combined and alone in choroidal neovascularization due to age-related macular degeneration. Ophthalmology 114: 1179-1185, 2007.

33. Datseris I, Kontadakis GA, Diamanti R, Datseris I, Pallikaris IG, Theodossiadis $\mathrm{P}$ and Tsilimbaris MK: Prospective comparison of low-fluence photodynamic therapy combined with intravitreal bevacizumab versus bevacizumab monotherapy for choroidal neovascularization in age-related macular degeneration. Semin Ophthalmol 30: 112-117, 2015.

34. Potter MJ, Claudio CC and Szabo SM: A randomised trial of bevacizumab and reduced light dose photodynamic therapy in age-related macular degeneration: the VIA study. Br J Ophthalmol 94: 174-179, 2010.

35. Saviano S, Leon PE, Mangogna A and Tognetto D: Combined therapy (intravitreal bevacizumab plus verteporfin photodynamic therapy) versus intravitreal bevacizumab monotherapy for choroidal neovascularization due to age-related macular degeneration: A 1-year follow-up study. Digit J Ophthalmol 22: 46-53, 2016.

36. Jadad AR, Moore RA, Carroll D, Jenkinson C, Reynolds DJ, Gavaghan DJ and McQuay HJ: Assessing the quality of reports of randomized clinical trials: Is blinding necessary? Control Clin Trials 17: 1-12, 1996.

37. Nowak-Sliwinska P, Van den Bergh H, Sickenberg M and Koh AH: Photodynamic therapy for polypoidal choroidal vasculopathy. Prog Retin Eye Res 37: 182-199, 2013.

38. Dabkeviciene D, Sasnauskiene A, Leman E, Kvietkauskaite R, Daugelaviciene N, Stankevicius V, Jurgelevicius V, Juodka B and Kirveliene V: mTHPC-mediated photodynamic treatment up-regulates the cytokines VEGF and IL-1alpha. Photochem Photobiol 88: 432-439, 2012.

This work is licensed under a Creative Commons Attribution-NonCommercial-NoDerivatives 4.0 International (CC BY-NC-ND 4.0) License. 